\title{
Educação permanente como estratégia de promoção da saúde mental para docentes
}

\section{universitários}

\author{
Continuing education as a mental health promotion strategy for university professors \\ La educación continua como estratégia de promoción de la salud mental para professores \\ universitarios
}

Recebido: 16/06/2021 | Revisado: 21/06/2021 | Aceito: 22/06/2021 | Publicado: 09/07/2021

\author{
Natânia Candeira dos Santos \\ ORCID: https://orcid.org/0000-0002-8168-957X \\ Universidade Federal Fluminense, Brasil \\ E-mail: n.candeira@gmail.com \\ Elaine Antunes Cortez \\ ORCID: https://orcid.org/0000-0003-3912-9648 \\ Universidade Federal Fluminense, Brasil \\ E-mail: nanicortez@hotmail.com
}

\begin{abstract}
Resumo
Essa pesquisa tem como objeto de estudo a Educação Permanente em Saúde como estratégia de promoção da saúde mental para docentes de magistério superior em uma Universidade Federal no estado do Rio de Janeiro. Tem como objetivo geral utilizar a Educação Permanente com professores de magistério superior na elaboração de processos e produtos de ensino como estratégia facilitadora para a promoção da saúde mental. Tratar-se-á de um estudo com método descritivo e exploratório, de abordagem quanti-qualitativa, do tipo pesquisa convergente assistencial, com aplicação de questionário semiestruturado e realização de oficinas com Metodologia de Problematização pelo Arco de Maguerez com os docentes da Escola de Enfermagem de uma Universidade Federal. Pretende-se discutir os dados obtidos à luz da Política Nacional de Educação Permanente e a partir dos referenciais teóricos de Paulo Freire, Política Nacional de Promoção da Saúde e da Saúde Mental Positiva. Espera-se que este estudo contribua para construir ferramentas que possibilitem promover saúde mental, bem como nas discussões com os profissionais sobre os possíveis impactos que o trabalho pode gerar na saúde mental, tornando-os mais conscientes da necessidade do autocuidado na melhoria da qualidade de vida.
\end{abstract}

Palavras-chave: Docentes; Saúde mental; Promoção da saúde; Ensino.

\begin{abstract}
This research has as its object of study the Permanent Education in Health as a strategy to promote mental health for professors of higher education at a Federal University in the state of Rio de Janeiro. Its general objective is to use Continuing Education with higher education teachers in the development of teaching processes and products as a facilitating strategy for the promotion of mental health. This will be a study with a descriptive and exploratory method, with a quanti-qualitative approach, of a convergent care research type, with application of a semi-structured questionnaire and workshops with Problematization Methodology by the Arch of Maguerez with teachers from the School of Nursing of a Federal University. It is intended to discuss the data obtained in the light of the National Policy for Continuing Education and from the theoretical references of Paulo Freire, National Policy for the Promotion of Health and Positive Mental Health. It is expected that this study will contribute to building tools that enable the promotion of mental health, as well as discussions with professionals about the possible impacts that work can generate on mental health, making them more aware of the need for self-care to improve the quality of life.
\end{abstract}

Keywords: Faculty; Mental health; Health promotion; Teaching.

\section{Resumen}

Esta investigación tiene como objeto de estudio la Educación Permanente en Salud como estrategia de promoción de la salud mental para profesores de educación superior de una Universidad Federal del estado de Río de Janeiro. Su objetivo general es utilizar la Educación Continuada con docentes de educación superior en el desarrollo de procesos y productos docentes como estrategia facilitadora para la promoción de la salud mental. Se trata de un estudio con método descriptivo y exploratorio, con enfoque cuanti-cualitativo, de tipo investigación asistencial convergente, con aplicación de cuestionario semiestructurado y talleres con Metodología de Problematización por el Arco de Maguerez con docentes de la Escuela de Enfermería de una Universidad Federal. Se pretende discutir los datos obtenidos a la luz de la Política Nacional de Educación Continuada y de los referentes teóricos de Paulo Freire, Política Nacional de Promoción de la Salud y Salud Mental Positiva. Se espera que este estudio contribuya a construir herramientas que 
permitan la promoción de la salud mental, así como discusiones con los profesionales sobre los posibles impactos que puede generar el trabajo en la salud mental, haciéndolos más conscientes de la necesidad del autocuidado para mejorar. la calidad de vida.

Palabras clave: Facultad; Salud mental; Promoción de la salud; Enseñanza.

\section{Introdução}

A atividade laboral do docente de magistério superior tem sofrido alterações ao longo dos anos junto aos avanços nos processos de globalização e de expansão universitária, os quais agiram aproximando essas instituições aos interesses de mercado impactando diretamente no fazer docente, que tem sido afetado pela forte intensificação na atuação desses profissionais (Guimarães \& Chaves, 2015).

Pesquisas sobre o trabalho docente têm demonstrado continuamente que esta categoria tem sido afetada grandemente pelo adoecimento mental, em que patologias relacionadas aos transtornos mentais e comportamentais têm sido contempladas como as maiores queixas de saúde e também de índices de afastamento no ambiente de trabalho, gerando sofrimento e sendo um importante problema de saúde pública (Campos et al., 2020; Farci et al., 2018; Guimarães \& Chaves, 2015).

De modo peculiar, o trabalho docente é retratado pelo excesso de atribuições e produtos, em que as demandas laborais ultrapassam os muros da universidade, e culminam na dedicação constante para ser suporte ao aluno e de produção científica. As exigências exorbitantes tendem a gerar sentimentos de preocupação, ansiedade, inquietação e aumento do estresse. Tais fatores vinculados à precarização do trabalho, prolongamento da jornada e sobrecarga reconfiguram os modos de trabalhar e influenciam na saúde e no bem-estar dos indivíduos (Rodrigues et al., 2020).

Por vezes, a estrutura organizacional do serviço impede a promoção da saúde, colocando os profissionais em um risco maior para o adoecimento. Reafirma-se que as novas organizações do trabalho têm caminhado transformando o conhecimento em mercadoria, principalmente pela busca incessante de produtividade desmedida. Não é incomum trabalhadores docentes queixando-se de sofrimento mental, evidenciado pela falta de perspectiva em descanso e lazer. Logo, é necessário que se lute contra a invasão do excesso de trabalho na vida pessoal, evitando os meios dominantes e de controle, prezando pela autonomia, liberdade e saúde mental (Rodrigues et al., 2020).

Pensando nos aspectos de saúde, tem-se que habitualmente, o modelo de saúde biomédico foi dominante em sua perspectiva fragmentada, centralizada e individualizada, havendo necessidade de mudanças desde as intenções com a Reforma Sanitária, as Cartas de Promoção da Saúde e o pensar ao modo do Sistema Único de Saúde (SUS), em que sujeitos e coletividades fossem engrenagem viva na construção da própria vida. Neste sentido, é possível compreender que a saúde vai além da instalação de uma doença, em que a qualidade de vida está ligada à expectativa e a um processo interligado entre as condições físicas, emocionais, econômicas e sociais (Brasil, 2014; Wilberstaedt et al., 2016).

As ligações entre as diversas condições e a construção da própria vida está presente, por exemplo, nas questões relativas ao autocuidado, uma vez que este é colocado com um dos aspectos para o viver saudável, sendo uma perspectiva em que o sujeito se coloca como responsável por suas ações, entendendo que tais ações estão ligadas ao seu cotidiano e às suas experiências vividas, mas que para além, torna-se também uma prática social quando existem trocas e relações interindividuais que podem agir de maneira promotora da saúde (Souza et al., 2014; Peláez \& Escobar, 2015).

Neste sentido, a Lei nº 8.080, de 19 de setembro de 1990 (Lei Orgânica da Saúde) trouxe a conceituação da saúde como uma consequência dos modos de vida e do contexto histórico, social, cultural, pensando para além dos aspectos meramente biológicos, incorporando a promoção da saúde como imprescindível para que se busque e compreenda as singularidades, a autonomia e a participação dos indivíduos em seus processos (Brasil, 2015).

Assim, através da Portaria no 2.446, de 11 de novembro de 2014, a Política Nacional de Promoção da Saúde (PNPS) foi reorientada trazendo como valores fundamentais em seu processo de efetivação: a solidariedade; a felicidade no sentido da 
autopercepção de satisfação; a ética; o respeito às diversidades; a humanização; a corresponsabilidade; a justiça social e a inclusão social. A PNPS objetiva de maneira geral "promover equidade e a melhoria das condições de viver, ampliando as potencialidades da saúde individual e da saúde coletiva, reduzindo vulnerabilidades e riscos à saúde decorrentes dos determinantes sociais, econômicos, políticos, culturais e ambientais" (Brasil, 2014).

Dessa forma, é possível repensar práticas e discutir realidades a partir da Educação Permanente em Saúde (EPS), uma vez que realizar a aproximação da vida cotidiana com a educação é possibilitar uma análise reflexiva dos problemas da prática laboral. No que diz respeito à organização do trabalho, os processos educativos podem propor uma análise baseada nas rotinas, normas, interações e tudo que gere vínculo, para que assim se possa transformá-los (Brasil, 2009).

Naturalmente, aborda-se o processo de reflexão das práticas como parte imprescindível para as mudanças organizacionais, fazendo com que seja possível uma equipe buscar pela reformulação de contextos reais que devem ser levados em consideração para análise (Brasil, 2009), neste caso, o contexto da saúde mental e da promoção da saúde mental no trabalho. Avanços devem ser validados e não reduzidos aos modelos anteriores, e por isso, é importante o engajamento e a articulação do trabalho com a educação permanente (Brasil, 2009) e aqui, acrescentando a promoção da saúde.

O tema dessa pesquisa se faz presente de forma potente e indispensável, apresentando como objeto de estudo a Educação Permanente em Saúde como estratégia de promoção da saúde mental para docentes de magistério superior em uma Universidade Federal no estado do Rio de Janeiro.

Por fim, pretende-se como objetivo geral utilizar a Educação Permanente com professores de magistério superior na elaboração de processos e produtos de ensino como estratégia facilitadora para a promoção da saúde mental, e com os objetivos específicos: avaliar a saúde mental positiva dos docentes; identificar as estratégias utilizadas pelos docentes para a promoção da saúde mental; elaborar, junto aos trabalhadores, processos/produtos de ensino que sensibilizem para a promoção da saúde mental.

\section{Metodologia}

A pesquisa terá como método a ser aplicado o descritivo e exploratório, de abordagem quanti-qualitativa, do tipo pesquisa convergente assistencial (PCA), tendo em vista que está orientada com um "compromisso humanista do pesquisador em estudar e operar na prática [...] a partir das perspectivas dos profissionais envolvidos no processo" (Trentini, 2014). Dessa forma, propõe o estudo e consequentemente o desenvolvimento de conhecimentos que estejam pautados na minimização dos problemas e na inovação, buscando do pesquisador sua imersão naquele campo de atuação (Trentini, 2014).

Junto a isso, a convergência na PCA diz respeito às ações de pesquisa e de assistência que se entrecruzam, sendo esse processo imprescindível na construção da base teórica e filosófica desse tipo de pesquisa (Trentini et al., 2017).

A pesquisa será realizada na Escola de Enfermagem da Universidade Federal, que funciona desde a década de 40, formando bacharéis e licenciados em enfermagem e tem como missão atuar na formação de profissionais a partir de atividades de ensino teórico, teórico-prático e estágio, de pesquisa, graduação, pós-graduação e de extensão, favorecendo o avanço científico e tecnológico da profissão de enfermagem.

A população convidada ao estudo será composta pelos docentes da referida escola, atribuindo-lhes como critérios de inclusão o vínculo empregatício estatutário estando em desenvolvimento pleno das suas funções na docência. Em contrapartida, devem ser excluídos da pesquisa aqueles que estejam licenciados, em período de férias ou cedidos a outras instituições no momento em que a coleta de dados for realizada. Ademais, a exclusão se estende àqueles que estejam envolvidos nesse estudo como orientador, coorientador e membros da banca.

A pesquisa está inserida no Programa de Pós-graduação do Mestrado Profissional de Ensino na Saúde da Universidade Federal Fluminense (MPES/UFF). O projeto foi previamente aprovado pela unidade a ser pesquisada através da 
assinatura do termo de anuência e encaminhado para aprovação pelo Comitê de Ética em Pesquisa (CEP) do Hospital Universitário Antônio Pedro (HUAP/UFF), através da Plataforma Brasil, dentro dos prazos estabelecidos pelo cronograma.

Este estudo segue atento às normas de pesquisas com Seres Humanos, mantendo-se pautado nas diretrizes e normativas regulamentadoras determinadas pela Resolução CNS nº. 466/2012 (Brasil, 2013), sendo ofertada e esclarecida a importância da participação através de conversa entre participante e pesquisadora, leitura conjunta do Termo de Consentimento Livre e Esclarecido - TCLE e sua assinatura conforme Resolução nº 510/2016 (Brasil, 2016).

Será garantida a confidencialidade e a privacidade daqueles que desejarem participar; a proteção da imagem; a não estigmatização e a não utilização das informações para prejuízo da pessoa. A preservação da identidade ocorrerá através da utilização de códigos por letras do alfabeto português e números arábicos (exemplo: P1, P2), além da proteção dos dados através de arquivo digital criptografado e arquivado em computador pessoal da pesquisadora. Após garantir o entendimento do que se trata a pesquisa e a extrema importância da participação para os campos de formação profissionais e também pessoais, o indivíduo estará ciente que poderá se retirar do estudo a qualquer momento sem que haja prejuízo algum.

Os depoimentos, reflexões e discussões serão gravados por meio de aparelho digital, com consentimento prévio dos partícipes, e posteriormente serão transcritos na íntegra para análise. É importante afirmar que haverá atenção para as necessidades de assistência integral decorrentes de qualquer dano físico, psíquico, moral, intelectual, social, cultural ou espiritual, mesmo que mínimos, mas que venha a ser gerado através da pesquisa.

A pesquisadora, a instituição e os participantes não receberão nenhuma vantagem financeira para a realização desta pesquisa, e não haverá necessidade de nenhum curso prévio ou qualquer capacitação para participação no estudo, sendo necessário apenas o cumprimento dos critérios de inclusão e exclusão pré-estabelecidos, assinatura do TCLE e do Termo de Concessão de Imagem.

$\mathrm{Na}$ tentativa de caracterizar os participantes foi elaborado, junto a orientadora, um questionário semiestruturado que respeitasse o tamanho da amostra; atentasse para a redação das questões; a forma de análise dos dados; margem de erro; e o processo de seleção dos participantes com a finalidade de enriquecer a discussão e reflexão para a temática abordada. Para avaliação da saúde mental positiva será utilizado o questionário de saúde mental positiva de Lluch-Canut (Lluch-Canut \& Sequeira, 2020).

Por conseguinte, acontecerão as oficinas de maneira a possibilitar o encontro entre os participantes, entendendo que devido à pandemia por COVID-19, tanto o questionário como as oficinas podem acontecer de maneira on-line, sendo discorrido de maneira clara no TCLE sobre as orientações para pesquisa em período de pandemia por COVID-19.

Para o momento dedicado às oficinas, é pretendido que aconteçam virtualmente, tendo em vista o momento atual da pandemia por COVID-19. É previsto que aconteçam seis encontros com pelo menos 1 hora de duração com a participação e interação entre os docentes, através de plataforma virtual gratuita do tipo google meet ou zoom, sem qualquer custo, exposições ou prejuízos para estes profissionais. A metodologia a ser utilizada será a Problematização pelo Arco de Maguerez com o uso de metodologias ativas de ensino-aprendizagem para estimular os debates.

Tem-se como primeira etapa do arco de Maguerez a observação da realidade em que questões gerais são propostas para guiar esse olhar, permitindo que haja identificação de problemas, conflitos, erros e tudo mais que um indivíduo pode se deparar quando encara aquele meio que ele está inserido. Os participantes devem criar um problema a partir dos fatores observados, justificando os motivos dessa escolha, colocando-se em uma postura crítica e reflexiva (Villardi, Cyrino \& Berbel, 2015).

Após a observação, os participantes devem traçar pontos-chave, refletindo e questionando possíveis determinantes para o problema encontrado, e assim, sendo possível compreender mais profundamente a questão, escolhendo tópicos que seja necessário investigar. Posteriormente, é o momento de teorização, em que a investigação de fato ocorrerá. Faz-se necessário 
buscar conhecimento e essa construção será a base para transformar a realidade, pois aqui adquire-se maior consciência sobre a problemática (Villardi, Cyrino \& Berbel, 2015).

Por conseguinte, chega o momento de construir hipóteses de solução instigando a capacidade reflexiva do grupo, pensando nas possibilidades que devem ser projetadas com base na etapa anterior, culminando nos alicerces para a obtenção de resultados frente ao problema. É preciso que se questione sobre o que pode ser feito para solucionar ou minimizar as questões apontadas (Villardi, Cyrino \& Berbel, 2015).

Finalmente, o caminhar pelo Arco de Maguerez faz chegar à aplicação prática à realidade, oportunidade em que se escolhe as propostas de solução mais viáveis que podem contribuir para superar o problema ou pelo menos parte dele. É uma etapa decisória, carregada de compromisso com o processo transformador, que garanta uma aplicação concreta de tudo que foi discutido e estudado até o momento, possibilitando a socialização do conhecimento (Villardi, Cyrino \& Berbel, 2015).

Problematizar poderá permitir que os profissionais, a partir de uma visão crítica, observem a realidade em que atuam e se manifestem sobre ela, transformando-a (Berbel, 2012). Escolher a utilização do Arco de Maguerez como metodologia a ser utilizada nesse estudo veio da necessidade de refletir sobre os problemas encontrados nesse ambiente laboral, o qual se encontra dotado de prática pedagógica e consequentemente social, que reverbera para além dos muros do trabalho. Está sendo proposto um trabalho ativo, participativo, reflexivo e crítico (Berbel \& Gamboa, 2011).

Importante ressaltar que essa metodologia pode fornecer suporte para buscar respostas aos problemas mesmo que estes sejam de um nível de complexidade maior. As discussões levantadas, seguindo-se o passo a passo do arco, podem melhorar a compreensão dos fatores facilitadores do adoecimento docente, estabelecendo diálogos para que se observe as diferentes perspectivas sobre a situação. Pode-se operar rompendo paradigmas, desconstruindo certezas à luz da investigação e promovendo posturas mais questionadores que por vezes foram deixadas à espera devido às sobrecargas de trabalho (Villardi, Cyrino \& Berbel, 2015; Silva et al., 2020).

Finalmente, as oficinas tendem a aproximar sujeitos, demandas e participação, sendo um importante espaço de convivência (Silva \& Malfitano, 2021). Desse modo, o conhecimento vai sendo estruturado pela troca e recepção de novas informações, fazendo com que haja interação entre conceitos, conteúdos e atribuindo significados que por vezes ainda não estavam presentes nas concepções de um ou outro indivíduo. (Ausubel, 2003).

Pretende-se analisar e discutir os dados obtidos à luz da Política Nacional de Educação Permanente e a partir dos referenciais teóricos de Paulo Freire, Política Nacional de Promoção da Saúde e da Saúde Mental Positiva.

\section{Resultados Esperados}

Espera-se que este estudo contribua para construir ferramentas que possibilitem promover saúde mental, bem como nas discussões com os profissionais sobre os possíveis impactos que o trabalho pode gerar na saúde mental, tornando-os mais conscientes da necessidade do autocuidado na melhoria da qualidade de vida. Acredita-se que agir junto aos trabalhadores na implementação da EPS nesse espaço, pode atuar no fortalecimento da universidade como ambiente promotor de bem-estar, inexoravelmente atrelada à consolidação de políticas do Sistema Único de Saúde (SUS), uma vez que este tem como pilar sensível a promoção da saúde.

Ademais, quanto à pesquisa científica irá colaborar na linha de pesquisa educação permanente no SUS no que se refere aos processos educativos dos profissionais como protagonistas da produção de saberes.

\section{Referências}

Ausubel, D. P. (2003). Aquisição e retenção de conhecimentos: uma perspectiva cognitiva. (1a. ed.): Plátano. 
Berbel, N. A. N., \& Gamboa, S. A. S. (2011). A metodologia da problematização com o Arco de Maguerez uma perspectiva teórica e epistemológica. Filosofia e Educação, 3(2), 264-287. https://periodicos.sbu.unicamp.br/ojs/index.php/rfe/article/view/8635462.

Brasil. (2009). Política Nacional de Educação Permanente em Saúde: Ministério da Saúde.

Brasil. (2013, 13 junho). Resolução nº 466, de 12 de dezembro de 2012. Dispõe sobre diretrizes e normas regulamentadoras de pesquisas envolvendo seres humanos.

Brasil. (2014, 11 novembro). Portaria Nº 2.446, de 11 de novembro de 2014. Redefine a Política Nacional de Promoção da Saúde (PNPS).

Brasil. (2015). Política Nacional de Promoção da Saúde: PNPS: revisão da Portaria MS/GM nº 687, de 30 de março de 2006. Brasília: Ministério da Saúde.

Brasil. (2016) Ministério da Saúde. Conselho Nacional de Saúde. Resolução nº 510, de 07 de abril de 2016. Normas aplicáveis a Pesquisas em Ciências Humanas e Sociais.

Campos, T. C., Véras, R. M., \& Araújo, T. M. (2020) Trabalho docente em universidades públicas brasileiras e adoecimento mental: uma revisão bibliográfica. Rev Doc Ens Superior, 10, e015193. https://periodicos.ufmg.br/index.php/rdes/article/view/15193.

Facci, M. G. D., Urt, S. C., \& Barros, A. T. F. (2018). Professor readaptado: a precarização do trabalho docente e o adoecimento. Rev Psic Escolar e Educacional, 22(2), 281-290. http://dx.doi.org/10.1590/2175-3539201802175546.

Guimarães, N. R., \& Chaves, V. L. J. (2015) A intensificação do trabalho docente universitário: aceitações e resistências. Rev Bras de Polit Adm da Educação, 31(3), 567-586 https://seer.ufrgs.br/rbpae/article/view/59914/37019.

Lluch-Canut, T., \& Sequeira, C. (2020). Saúde mental positiva. In C. Sequeira, \& F. Sampaio (Org.), Enfermagem em saúde mental: diagnósticos e intervenções (pp. 61-63). Lidel.

Peláez, Z. R. F., \& Escobar, J. A. D. (2015) La bioética y el autocuidado de la salud: imperativos para la formación integral en la universidad. Acta bioeth, 21(1), https://scielo.conicyt.cl/scielo.php?pid=S1726-569X2015000100005\&script=sci_arttext\&tlng=e.

Rodrigues, A. M. S., Souza, K. R., Teixeira, L. R., \& Larentis, A. L. (2020) A temporalidade social do trabalho docente em universidade pública e a saúde. Ciência \& Saúde Coletiva, 25(5), 1829-1838. https://doi.org/10.1590/1413-81232020255.3322201.

Silva, M. J., \& Malfitano, A. P. S. (2021). Oficinas de atividades, dinâmicas e projetos em Terapia Ocupacional Social como estratégia para a promoção de espaços públicos. Interface, 25, e200055. https://doi.org/10.1590/interface.200055.

Silva, A. N., Senna, M. A. A., Teixeira, M. C. B., Lucietto, D. A., \& Andrade, I. M. (2020). O uso de metodologia ativa no campo das Ciências Sociais em Saúde: relato de experiência de produção audiovisual por estudantes. Interface, 24, e190231. https://doi.org/10.1590/Interface.190231.

Souza, M. C. B., Salomon, A. S. C., \& Lima, B. E. R. (2014) A prática do autocuidado pelo profissional docente enfermeiro. Revista Eletrônica Gestão \& Saúde, 05(2), 290-02. https://periodicos.unb.br/index.php/rgs/article/download/429/412/849.

Trentini, M. (2014). Pesquisa Convergente Assistencial-PCA: delineamento provocador de mudanças nas práticas de saúde. (3.a. ed.). Porto Alegre: Moriá.

Trentini, M., Paim, L., \& Silva, D. M. G. V. (2017). O método da pesquisa convergente assistencial e sua aplicação na prática de enfermagem. Texto Contexto Enferm, 26(4), e1450017. https://doi.org/10.1590/0104-07072017001450017.

Villardi, M. L., Cyrino, E. G., \& Berbel, N. A. N. (2015). A metodologia da problematização no ensino em saúde: suas etapas e possibilidades. In: [online]. São Paulo: Editora UNESP, (pp. 45-52). ISBN 978-85-7983-662-6. http://books.scielo.org.

Wilberstaedt, I. O. S., Vieira, M. G. M., \& Flores e Silva, Y. (2016). Saúde e qualidade de vida: discursos de docentes no cotidiano de uma escola pública de Santa Catarina. Trab. Educ. Saúde, 14, supl .1, 219- 238. http://dx.doi.org/10.1590/1 981-7746-sol00026. 